\title{
Ofloxacin compared with chloramphenicol in the management of external ocular infection
}

\author{
A J Bron, G Leber, S N M Rizk, H Baig, A R Elkington, G R Kirkby, C Neoh, A Harden, \\ T Leong
}

\begin{abstract}
The safety and efficacy of $0.3 \%$ ofloxacin in treating bacterial ocular infections was compared with that of $0.5 \%$ chloramphenicol in a parallel-group, randomised clinical trial at five sites. Clinical and microbiological improvement rates were studied in 84 culture-positive patients. Patients with suspected bacterial ocular infections were evaluated for clinical improvement and were included in drug safety and comfort analyses. Clinical improvement did not differ significantly between drug treatments. All patients completing the study (79 assigned ofloxacin, and 74 chloramphenicol) showed clinical improvement. Clinical improvement in the culture-positive groups was $100 \%(41 / 41)$ after ofloxacin treatment, and $95 \%(41 / 43)$ after chloramphenicol treatment. Microbiological improvement rates were similar for the two drugs: $85 \%(33 / 39)$ improved with ofloxacin, and $88 \%(38 / 43)$ improved with chloramphenicol. Both drugs were well tolerated. Adverse reactions possibly due to the study medication occurred in $1 \%(1 / 89)$ of those who received ofloxacin, and in $4 \%(4 / 93)$ of those who received chloramphenicol.
\end{abstract}

Bacterial infection of the outer eye is common, but there is a limited range of antimicrobial agents available for topical therapy. The search for new agents is influenced by the emergence of resistant bacterial strains to some topical preparations and by considerations of local and systemic toxicity. ofloxacin, a new antibiotic, was compared with that of chloramphenicol, an agent widely used against external bacterial ocular infections. This comparison was undertaken because chloramphenicol resistance has become established in several pathogens. ${ }^{12}$ Moreover, rare but serious reactions have been associated with topical ocular use of chloramphenicol. ${ }^{3-5}$

Ofloxacin, a synthetic fluorinated quinolone first described in 1982, shows promise as an

Accepted for publication 2 May 1991
In the following study the clinical efficacy of

agent against external ocular infections. ${ }^{6}$ Ofloxacin is exceptionally potent against a wide range of Gram-positive and Gram-negative bacteria and obligate anaerobes. ${ }^{78}$ It is effective in vitro against many organisms that cause ocular infections (Table 1), for example Haemophilus influenzae, Neisseria gonorrhoeae, Staphylococcus species and Streptococcus species. ${ }^{910}$ Ofloxacin also acts against Pseudomonas aeruginosa, ${ }^{1}$ which is associated with ocular infections that are especially serious and difficult to treat.

Quinolones are bactericidal in mode of action; they specifically inhibit the bacterial DNA gyrase supercoiling function required by bacterial, but not eukaryotic cells. ${ }^{12}$ In vivo, ofloxacin performs better than other fluoroquinolones with similar in vitro $\mathrm{MIC}_{90}$ values (minimum concentration that inhibits $90 \%$ of isolates in vitro). ${ }^{13}$ The pharmacokinetic indications $^{14}$ is that it will be an effective topical ocular antibiotic have also been confirmed by clinical tests. ${ }^{16}$ Chloramphenicol, isolated from Streptomyces venezuelae in 1947, has been an effective agent against external ocular infections for about 40 years. ${ }^{17}$ Chloramphenicol acts by inhibiting prokaryotic protein synthesis. ${ }^{18}$ The action of chloramphenicol is usually bacteriostatic, but it is bactericidal against Haemophilus influenzae, Streptococcus pneumoniae, and Neisseria meningitidis. ${ }^{19}$

\section{Patients and methods}

This double-masked, parallel-group, clinical trial was conducted at five sites. Selected patients with suspected bacterial ocular infection were randomly assigned either $0.3 \%$ ofloxacin or $0.5 \%$ chloramphenicol ophthalmic solution for a seven-day treatment.

\section{PATIENTS}

Patients were screened for several conditions. Other ocular disease, contact lens wear, and use of other ophthalmic medications or of systemic antibiotics were grounds for exclusion. Patients sensitive to chloramphenicol or to any quinolone were excluded. Those with uncontrolled systemic disease and pregnant or nursing women were not enrolled. Informed consent was obtained from all enrolled patients. agents against five major ocular pathogens, expressed as the MIC concentration against $90 \%$ of all bacterial strains tested. ${ }^{.1}$

\begin{tabular}{llllc}
\hline \multirow{4}{*}{$M I C(\mu \mathrm{g} / \mathrm{ml})$} & & \\
\cline { 2 - 5 } Organism (no) & Ofloxacin & Gentamicin & Tobramycin & Chloramphenicol \\
\hline Haemophilus influenzae (18) & 4 & $3 \cdot 4$ & 2 & 20 \\
Pseudomonas aeruginosa (68) & 4 & 8 & $4 \cdot 4$ & 128 \\
Staphylococcus aureus (79) & $0 \cdot 5$ & 1 & 2 & 16 \\
Staphylococcus epidermidis (68) & $0 \cdot 5$ & 32 & 64 & 32 \\
Streptococcus pneumoniae (21) & 2 & 7 & 16 & 4 \\
\hline
\end{tabular}

\section{SCHEDULE OF VISITS AND MEASUREMENTS}

Symptoms were assessed by an ophthalmic examination (best corrected visual acuity, slitlamp biomicroscopy, and ophthalmoscopy) at visit 1 (day 1), visit 2 (days 3-5), and visit 3 (day 8). On day 1 ocular cultures were obtained from 


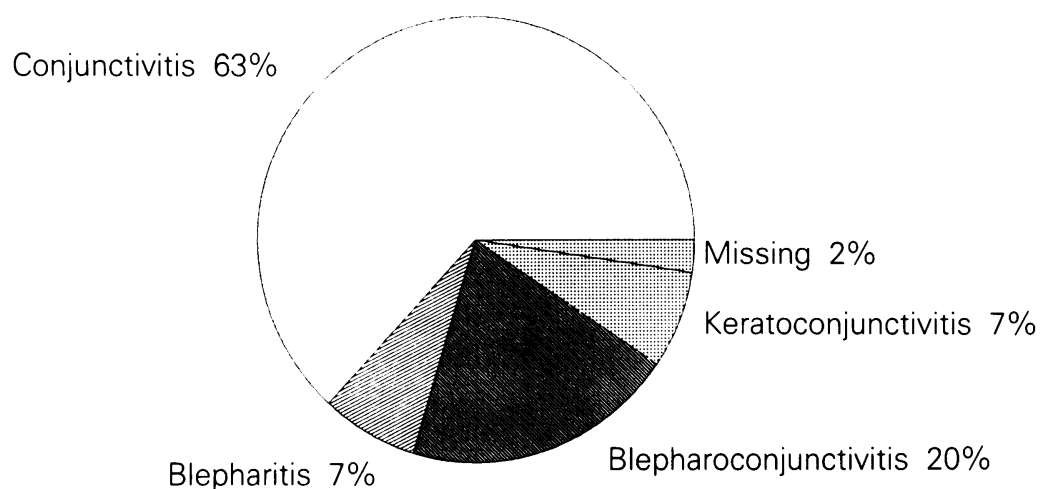

Figure 1 Distribution of diagnoses in 84 patients who entered the study with positive ocular microbiological tests. (Sum does not equal $100 \%$ owing to rounding error.) * One case of blepharokeratoconjunctivitis included under keratoconjunctivitis.

all patients as described below. Investigators applied the first drug dose, and patients were instructed to use their eye drops every 2-4 waking hours (according to severity as determined by the physician) on days 1 and 2 four times daily on days 3 through 7 , and to stop treatment at least 12 hours before visit 3 on day 8 .

Symptoms and drug tolerance were evaluated at visits 2 and 3 . A second ocular culture was taken on visit 3 from patients whose first culture had given a positive result.

\section{MICROBIOLOGICAL METHODS}

Ocular cultures were obtained by rolling the tip of a calcium alginate swab, moistened with sterile unpreserved saline, along the lid margin and conjunctival cul-de-sac of each affected eye. After the culture sample was obtained, each swab was placed in a sterile tube containing 1.0 $\mathrm{ml}$ of transport dissolving buffer solution (sodium citrate [dihydrate], sodium chloride, sodium acetate [trihydrate], potassium chloride, calcium chloride [dihydrate], magnesium chloride [hexahydrate], hydrochloric acid, sodium hydroxide, and purified water) and delivered to the microbiological laboratory within 4 hours for quantitative analysis.

On arrival at the laboratory the transport tube was vortexed until the swab fibres were finely dispersed. A sample of the suspension was directly plated on to a $5 \%$ horse blood agar plate (BAP) and a chocolate agar plate (CAP). A 1:10 dilution of the sample was prepared in sterile trypticase soy broth and also plated onto BAP and CAP. All sample plates were incubated for 48 hours at $37^{\circ} \mathrm{C}$ in $4 \% \mathrm{CO}_{2}$. Colony-forming units were counted, bacteria identified, and sensitivity tests to ofloxacin and chloramphenicol were performed.

\section{MICROBIOLOGICAL IMPROVEMENT}

Classification of cultures as positive or negative followed the methods and classification of bacterial thresholds of Cable et al. ${ }^{2021}$ A culture was considered positive if the colony count exceeded the threshold for any bacterial class. For class A, the most virulent ocular pathogens (group A streptococci, other $\beta$-haemolytic streptococci, $S$. pneumoniae, all Gram-negative rods and Neisseria species), the threshold was above zero colony-forming units. For class B, $\alpha$-haemolytic streptococci (other than S. pneumoniae), Staphylococcus aureus, micrococci, and Branhamella catarrhalis, the threshold was more than 10. For class C other Staphylococcus species (including Staphylococcus epidermidis) and Bacillus species, the threshold was more than 100 . The threshold of class $D$, Corynebacterium species, was more than 10000 colony-forming units.

Either eradication (zero colonies) or control of bacteria at visit 3 was scored as microbiological improvement. Bacteria were considered controlled if the colony count dropped below threshold values.

\section{CLINICAL IMPROVEMENT AND SAFETY}

Clinical improvement was defined as a decline in symptoms of external ocular infection. The composite severity score (CSS) of 10 factors (lid erythema, oedema and crusting, palpebral and bulbar conjunctival hyperaemia, conjunctival oedema and discharge, corneal oedema, foreign body sensation, and pain) was determined for all patients. Biomicroscopy and symptoms were graded 0 for none, 1 for mild, 2 for moderate, and 3 for severe.

Drug comfort was determined from reported irritation or pain following use of the drug. Safety was assessed by reported adverse reactions and by comparing visual acuity, lens pathology and ophthalmoscopy before and after treatment.

\section{OVERALL IMPROVEMENT}

Overall improvement, defined as showing both microbiological and clinical improvement at visit 3 , was determined for initially culture-positive patients.

\section{STATISTICAL ANALYSES}

Clinical, microbiological and overall improvement rates were compared by the CochranMantel-Haenszel (CMH) test $^{22}$ stratified by the investigator. The CSS of the treatment groups was compared by a two-way analysis of variance. For all analyses a $p$ value less than or equal to 0.05 was considered statistically significant.

\section{Results}

A total of 167 patients with suspected bacterial eye infections were enrolled for clinical efficacy and safety evaluations. Age ranged from 19 to 81 . On visit 1,83 patients received ofloxacin, 84 received chloramphenicol. Treatment groups did not differ significantly in age, race, sex, iris colour, medical or ophthalmic history.

The frequencies of each diagnosis were the same in both treatment groups. Two conditions accounted for most cases. In the culture-positive group (Fig 1), conjunctivitis was diagnosed in $63 \%(53 / 84)$ of the patients, and blepharoconjunctivitis in another $20 \%$ (17/84). The remaining diagnoses were distributed among the following: blepharitis, blepharokeratoconjunctivitis, and keratoconjunctivitis. Two diagnoses were not recorded. 


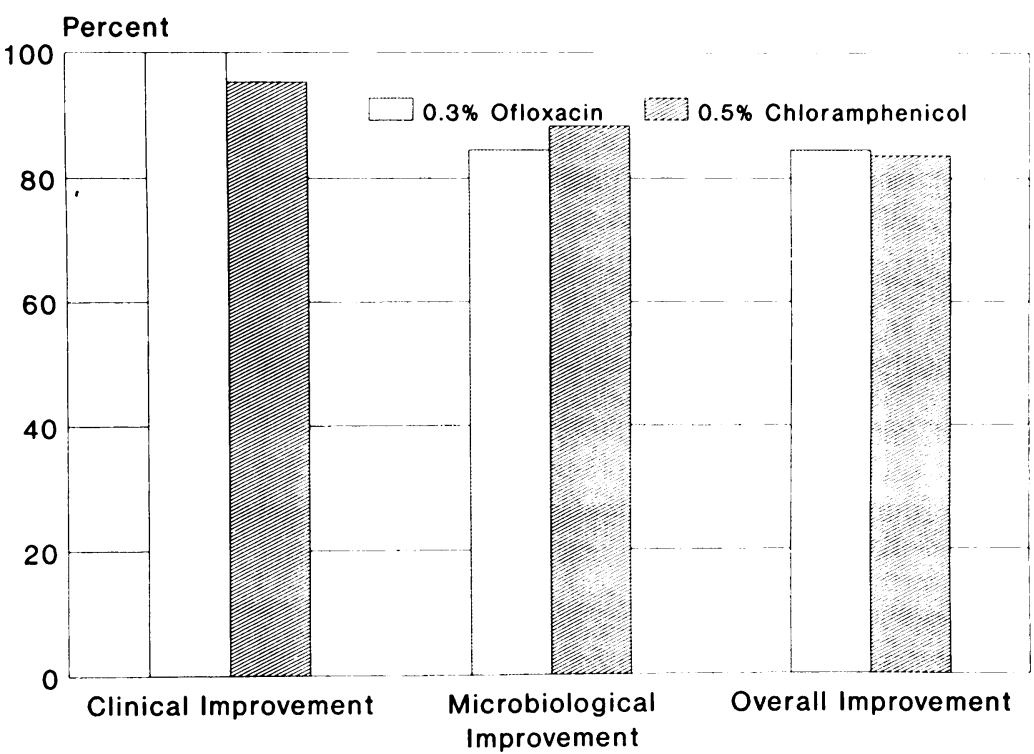

Figure 2 Clinical, microbiological, and overall improvement rates for initially culturepositive patients treated for seven days with ofloxacin or chloramphenicol.

MICROBIOLOGICAL ISOLATES

The culture-positive group comprised 84 patients who were microbiologically positive on day 1 . Culture records were completed for 39 of 41 culture-positive patients assigned ofloxacin, and for all 43 patients assigned chloramphenicol.

Of 32 Gram-negative organisms isolated from ocular cultures on day 1 none was resistant to ofloxacin, but one was resistant to chloramphenicol. Of 152 Gram-positive organisms tested, one was resistant to ofloxacin, and four were resistant to chloramphenicol.

IMPROVEMENT IN CULTURE-POSITIVE GROUP High improvement rates were obtained with both drugs. No statistically or clinically significant differences were found between treatments in microbiological, clinical, or overall improvement rates of the initial culture-positive group (Fig 2).

Microbiological improvement rates were $85 \%$ (33/39) for ofloxacin, and $88 \%$ (38/43) for chloramphenicol. Bacteria were eradicated by visit 3 in 79\% (31/39) of patients given ofloxacin and in $77 \%(33 / 43)$ of those given chloramphenicol.

The two drugs were similarly effective against all bacterial genera and threshold classes encountered in this trial. For conjunctivitis due to common causative organisms the microbiological improvement rates are shown in Table 2 .

Clinical improvement rates were $100 \%(41 / 41)$ for ofloxacin, and 95\% (41/43) for chloram-

Table 2 The microbiological improvement rates of patients after treatment with ofloxacin and chloramphenicol against organisms commonly associated with conjunctivitis

\begin{tabular}{|c|c|c|c|c|}
\hline \multirow[b]{3}{*}{ Organism } & \multicolumn{4}{|c|}{ Improvement rate } \\
\hline & \multicolumn{2}{|l|}{ Ofloxacin } & \multicolumn{2}{|c|}{ Chloramphenicol } \\
\hline & $\%$ of patients & (No of patients) & $\%$ of patients & (No of patients) \\
\hline Haemophilus influenzae & 100 & $11 / 11$ & 100 & $12 / 12$ \\
\hline Staphylococcus aureus & 96 & $26 / 27$ & 87 & $20 / 23$ \\
\hline Staphylococcus epidermidis & 88 & $23 / 26$ & 94 & $33 / 35$ \\
\hline Streptococcus sp. & 92 & $12 / 13$ & 100 & $29 / 29$ \\
\hline
\end{tabular}

phenicol. Among patients with conjunctivitis clinical improvement rates were $100 \%(27 / 27)$ for ofloxacin treatment, and $96 \%(25 / 26)$ for chloramphenicol treatment.

Overall improvement rates were 85\% (33/39) and $84 \%(36 / 43)$ for ofloxacin and chloramphenicol, respectively. Overall improvement in culture-positive patients with conjunctivitis was $88 \%(22 / 25)$ for ofloxacin treatment and $85 \%$ (22/ 26) for chloramphenicol treatment.

In six of the 11 unimproved patients the bacteria present on day 8 were not present on day 1. Among those using chloramphenicol different species were present in 5\% (2/43) (Staphylococcus capitis; Staphylococcus epidermidis) at visit 3 . New organisms were present in $10 \%(4 / 39)$ of those using ofloxacin (Micrococcus sp., Staphylococcus epidermidis, $\alpha$-haemolytic streptococci, and Staphylococcus aureus). In five unimproved patients the same bacteria increased above counts on day 1 in $13 \%(5 / 39)$ of patients treated with ofloxacin and in $9 \%(4 / 43)$ of those treated with chloramphenicol.

\section{BIOMICROSCOPY, SYMPTOMS AND SAFETY - ALL} PATIENTS

For all 167 patients there were no notable differences between treatment groups at day 1 . CCS declined after treatment with both drugs, and no significant difference in any symptom was present at visit 3 . No significant changes were detected by ophthalmoscopy during the study. No changes in visual acuity were correlated with any observed biomicroscopy changes.

There was no significant difference between groups in adverse reactions. Of 83 patients receiving ofloxacin, one reported a reaction described as haemorrhagic conjunctivitis and marked palpebral oedema which may have been drug induced. Four of 84 patients who received chloramphenicol left the study after various complaints (for example, atopic reaction; stinging and eye soreness; headache; lip ulceration) which were judged to be possibly due to the study medication. These five patients were discontinued from the clinical trial, and were not rechallenged with the study medication.

\section{Discussion}

The development of novel anti-infective agents is required by the evolution of bacterial drug resistance because the effectiveness of any antibiotic can be impaired by resistant pathogens. The relative effectiveness and safety of alternative treatments must be considered in choosing any course of therapy, but a comparison of effectiveness and risk of side effects is especially important in evaluating the potential of a new therapeutic agent.

Chloramphenical is a potent, broad-spectrum antibiotic, and is still widely prescribed for external ocular infection. Unfortunately two factors threaten to erode its usefulness: the appearance of resistant bacterial pathogens, and the recognition of sometimes serious side effects associated with chloramphenicol therapy. The imminent need for alternative treatments notwithstanding, a prospective replacement must 
match the high performance of chloramphenicol while posing fewer risks.

Our study confirms that ofloxacin is a safe new antimicrobial agent effective in treating external ocular infection. ${ }^{16}$ Ofloxacin performed as well as chloramphenicol in promoting microbiological, clinical, and overall improvement in patients with bacterial eye infections (Fig 2). Ofloxacin eradicated or controlled bacteria in $79 \%(31 / 39)$ of culture-positive patients compared with $76 \%$ (38/43) for chloramphenicol.

In vitro studies indicate that both ofloxacin and chloramphenicol are broad spectrum antibiotics. ${ }^{2324}$ However, the MIC $_{90}$ (minimum concentration that inhibits $90 \%$ of isolates in vitro) of ofloxacin is substantially lower than that of chloramphenicol for several important ocular pathogens. In fact, $P$. aeruginosa, a dangerous class A pathogen, is considered insensitive to chloramphenicol treatment. ${ }^{25}$ The MIC $_{90}$ for ofloxacin is $4 \mu \mathrm{g} / \mathrm{ml}$ compared with $128 \mu \mathrm{g} / \mathrm{ml}$ for chloramphenicol against $P$. aeruginosa." The $\mathrm{MIC}_{90}$ was $0.5 \mu \mathrm{g} / \mathrm{ml}$ for ofloxacin against both $S$. aureus and $S$. epidermidis, whereas the respective values were $16 \mu \mathrm{g} / \mathrm{ml}$ and $32 \mu \mathrm{g} / \mathrm{ml}$ for chloramphenicol."

Chloramphenicol resistance occurs in many pathogenic species, including $H$. influenzae and $S$. pneumoniae. High frequencies of resistance, ranging from $30 \%$ to $58 \%$ of clinical isolates, have been observed. ${ }^{26-28}$ The potential for rapid spread of chloramphenicol resistance is high because plasmid-borne factors exist, and conjugative transfer of the plasmid occurs. ${ }^{29-31}$ In contrast, ofloxacin-resistant strains seldom arise, ${ }^{32}$ and the development of plasmid-borne resistance is viewed as unlikely. ${ }^{33}$ Moreover, ofloxacin-resistant strains are typically slow growing and so unstable that they fail to compete with sensitive strains or revertants under ofloxacin-free conditions. ${ }^{34}$ Though the 184 isolates sampled in this study did not differ significantly in their resistance to the drugs, five were insensitive to chloramphenicol, and one was insensitive to ofloxacin in vitro.

Along with efficacy safety is of primary importance in comparing drug treatment. Chloramphenicol can cause rare, but serious, blood dyscrasias, and fatal aplastic anaemia occurs in about 1 in 40000 courses of systemic chloramphenicol therapy. ${ }^{35} 36$ Topical ocular use of chloramphenicol has also resulted in blood dyscrasias and deaths from aplastic anaemia, an idiosyncratic reaction that is independent of dose. . $^{3-537}$

Adverse reactions to systemic ofloxacin occur at low rates of $2 \%$ to $7 \%$ of treated patients. ${ }^{38}$ Clinical studies of 15962 patients yielded about four adverse reactions per 100 patients who were administered an oral form of ofloxacin; such reactions were typically mild gastrointestinal complaints or minor nervous symptoms. ${ }^{39} \mathrm{~A}$ low frequency, $0 \cdot 5 \%(38 / 7566)$, of adverse reactions to an ophthalmic ofloxacin preparation was found in a post-marketing study completed in 1989 by Santen Corp., Japan (communicated to Allergan Inc.).

Ofloxacin possesses outstanding potency against a variety of pathogens, and is active against strains resistant to chloramphenicol and other antibiotics..$^{+0-12}$ In comparing the utility of ofloxacin to that of chloramphenicol we must also consider the side effects of both drugs. Ofloxacin has negligible side effects when administered orally or topically. However, the risks associated with chloramphenicol have led some authors $\mathrm{s}^{43}$ to suggest using this antibiotic only when other agents fail. In considering effectiveness and safety, the clinical equivalence of ofloxacin compared with chloramphenicol has significance for the management of external bacterial ocular infections.

Funding for this study was provided by a grant from Allergan, Inc.

1 Kenny JF, Isburg CD, Michaels RH. Meningitis due to Haemophilus influenzae type $\mathrm{b}$ resistant to both ampicillin and chloramphenicol. Pediatrics 1980; 66: 14-6.

2 Radetsky MS, Istre GR, Johansen TL, et al. Multiply resistant Pneumococcus causing meningitis; its epidemiology within a day-care centre. Lancet 1981; ii: 771-3.

3 Abrams SM, Degnan TJ, Vinciguerra V. Marrow aplasia following topical application of chloramphenicol eye ointment. Arch Intern Med 1980; 140: 576-7.

4 Fraunfelder FT, Bagby GC, Kelly DJ. Fatal aplastic anemia following topical administration of ophthalmic chloramfollowing topical administration of ophthalmic

5 Brodsky E, Zeidan A, Biger Y, Schneider M. Topical application of chloramphenicol eye ointment followed by fatal bone marrow aplasia. Israel f Med Sci 1989; 25: 54.

6 Borrmann LR, Leopold IH. The potential use of quinolones in future ocular antimicrobial therapy. Am f Ophthalmol 1988; 106: $227-9$.

7 Sato K, Inoue Y, Fujii T, Aoyama H, Mitsuhashi S. Antibacterial activity of ofloxacin and its mode of action. Infection 1986; 14 (suppl 4): S226-30.

8 Speciale A, Stefani S, Caccamo R, Nicolosi VM, Nicoletti G. The sensitivity of Gram-negative and Gram-positive bacteria to ofloxacin. Drugs Exp Clin Res 1987; 12: 555-61.

9 King A, Shannon K, Phillips I. The in-vitro activities of enoxacin and ofloxacin compared with that of ciprofloxacin. F Antimicrob Chemother 1985; 15: 551-8.

10 Debbia E, Mannelli S, Gianrossi G, Schito GC. Susceptibility in vitro of gram-positive aerobe and anaerobe bacteria to ofloxacin. Drugs Exp Clin Res 1987; 13: 213-7.

11 Osato MS, Jensen HG, Trousdale MD, et al. The comparative in vitro activity of ofloxacin and selected ophthalmic antimicrobial agents against ocular bacterial isolates. Am f Ophthalmol 1989; 108: 380-6.

12 Kayser FH. The quinolones: mode of action and mechanism of resistance. Res Clin Forums 1985; 7: 17-24.

13 Chantot JF, Bryskier A. Antibacterial activity of ofloxacin and other 4-quinolone derivatives: in-vitro and in-vivo comparison. F Antimicrob Chemother 1985; 16: 475-84.

14 Borrmann L, Tang-Liu D, Kann J, Nista J, Akers P, Frank J. Tear levels and systemic absorption of ofloxacin eyedrops in humans. Invest Ophthalmol Vis Sci 1989; 30 (suppl): 247.

15 Monk JP, Campoli-Richards DM. Ofloxacin: a review of its antibacterial activity, pharmocokinetic properties and therapeutic use. Drugs 1987; 33: 346-91.

16 Mitsui Y, Sakuragi S, Tamura O, et al. Effect of ofloxacin ophthalmic solution in the treatment of external bacterial infections of the eye. Folia Ophthalmol fpn 1986; 37 1115-40.

17 Newman P. Macrolide and related antibiotics. In: Smolin G Okumoto MA, eds. Antimicrobial agents in ophthalmology. New York: Masson, 1981: chapter 10.

18 Shaw WV. Comparative enzymology of chloramphenico resistance. Ann New York Acad Sci 1971; 182: 234-42.

19 Rahal JJ, Simberloff MS. Bactericidal and bacteriostatic action of chloramphenicol against meningeal pathogens. Antimicrob Agents Chemother 1979; 16: 13-8.

20 Cagle GD, Abshire RL. Quantitative ocular bacteriology: method for the enumeration and identification of bacteria from the skin-lash margin and conjunctiva. Invest Ophthalmol Vis Sci 1981; 20:751-7.

21 Cagle GD, Davis S, Rosenthal A, Smith J. Topical tobramycin and gentamicin sulfate in the treatment of ocular infections: multicenter study. Curr Eye Res 1982; 1: 523-34

22 Landis RJ, Heyman ER, Koch GG. Average partial association on three-way contingency tables: a review and discussion of alternative tests. Int Statist Rev 1978; 46: 237-54.

23 Sato K, Matsuura Y, Inoue M, Une T, Osada Y, Ogawa $H$, Mitsuhashi S. In vitro and in vivo activity of DL-8280, new oxazine derivative. Antimicrob Agents Chemother 1982 22: 548-53.

24 Chang FW. Anti-infective drugs. In: Bartlett JD, Jaanus SD, 1984: 193-220.

25 Powell DA, Nahata MC. Chloramphenicol: new perspectives on an old drug. Drug Intellig Clin Pharm 1982; 16: 295-300. Mahajan VM. Acute bacterial infections of the eye: their aetiology and treatment. Brf Ophthalmol 1983; 67: 191-4.

27 French GL, Ling J, Ling T, Hui YW. Susceptibility of Hong Kong isolates of methicillin-resistant Staphylococcus aureus to antimicrobial agents. $\mathcal{F}$ Antimicrob Chemother 1988; 21: to antim. 
28 Dirdal M. Fucithalmic in acute conjunctivitis. Open, randomized comparison of fusidic acid, chloramphenicol and framycetin eye drops. Acta Ophthalmol $(K G h)$ 1987; 65: 129-33.

29 Roberts MC, Swensen C, Owens L, Smith AL. Characterization of chloramphenicol resistant Haemophilus influenzae. Antimicrob Agents Chemother 1980; 18: 610-5.

30 Pepper K, Le Bouguenec C, de Cespedes G, Horaud T Dispersal of a plasmid-borne chloramphenicol resistance gene in streptococcal and enterococcal plasmids. Plasmid 1986; 16: 195-203.

31 Van Klingeren B, Van Embden J, Dessens-Kroon $M$ Plasmid-mediated chloramphenicol resistance in Haemo-
philus influenzae. Antimicrob Agents Chemother 1977; 11: philus

32 Kresken $M$, Wiedemann B. Development of resistance to nalidixic acid and the fluoroquinolones after the introduction of norfloxacin and ofloxacin. Antimicrob Agents Chemother 1988; 32: 1285-8

33 Fernandes PB. Mode of action, and in vitro and in vivo activities of the fluoroquinolones. F Clin Pharmacol 1988; 28: $156-68$.

34 Crumplin GC, Odell $M$. Development of resistance to ofloxacin. Drugs 1987; 34 (suppl 1): 1-8.

35 Fraunfelder FT, Bagby GC Fatal aplastic anemia. FAMA 1982; 247: 2499

36 Wallerstein RO, Condit PK, Kasper GK. Statewide study of chloramphenicol therapy and fatal aplastic anemia. $\mathcal{F} A M A$ 1969; 208: 2045-50.

37 West BC, DeVault GA, Clement JC, Williams DM. Aplastic anemia associated with parenteral chloramphenicol: review of 10 cases, including the second case of possible increased risk with cimetidine. Rev Infect Dis 1988; 10: 1048-51.

38 Jungst $G$, Mohr R. Overview of postmarketing experience with ofloxacin in Germany. $\mathcal{F}$ Antimicrob Chemother 1988; 22 (suppl C): 167-75.

39 Junst G, Mohr R. Side effects of ofloxacin in clinical trials and in postmarketing surveillance. Drugs 1987; 34 (suppl 11): in post

40 Smith SM. In vitro comparison of A-56619, A-56620, amifloxacin, ciprofloxacin, enoxacin, and ofloxacin against methicillin-resistant Staphylococcus aureus. Antimicrob Agents Chemother 1986; 29: 325-6.

41 Kumada T, Neu HC. In-vitro activity of ofloxacin, a quinolone carboxylic acid compared to other quinolones and other microbial agents. 7 Antimicrob Chemother $1985 ; 16: 563-74$

42 Ridgway GL, O'Hare MD, Felmingham D, Gruneberg RN The comparative activity of twelve t-quinolone antimicrobials against Haemophilus influenzae and Streptococcus pneumatiae. Drugs Exp Res 1985; 11:259-62.

43 pneumatiae. Drugs Exp Res 1985; $11: 259-62$. decade. South Med f 1986; 79: 1129-34.

44 Fraunfelder FT, Meyer SM. Systemic reactions to ophthalmic drug preparations. Med Toxicol Adverse Drug Exp 1987; 2: 287-93. 